\title{
Bioaccumulation des métaux chez le mytilidae hydrothermal Bathymodiolus sp. de la ride médio-atlantique
}

\author{
Nathalie ROUSSE ${ }^{a}$, Jacques BOULEGUE ${ }^{a}$, Richard P. COSSON ${ }^{b}$, Aline FIALA-MEDIONI ${ }^{c}$ \\ ${ }^{a}$ Laboratoire de Géochimie et Métallogénie, Université Pierre et Marie Curie, 4, place Jussieu, 75252 Paris cedex 05 , \\ France \\ ${ }^{\mathrm{b}}$ EP 61-CNRS-ISOMER, 1, rue Gaston-Veil, 44035 Nantes, France \\ ${ }^{\mathrm{c}}$ Laboratoire Arago, Université Pierre et Marie Curie, B.P. 44, 66650 Banyuls-sur-Mer, France
}

(Received 11/06/97, revised 28/04/98, accepted 04/05/98)

\begin{abstract}
Bioaccumulation of metals within the hydrothermal mytilidae Bathymodiolus sp. from the Mid-Atlantic Ridge. The hydrothermal environment is characterised, among other things, by a high metal concentration, related to the convective sea-water circulation inside the oceanic crust and its interaction with basaltic rocks. The biological communities associated with the hydrothermal system can survive in this toxic environment owing to their ability to regulate their intracellular metal levels by excretion or accumulation of metal ions in non-toxic forms. Various detoxification processes have been previously studied within an hydrothermal organism: immobilisation and precipitation of the metal in lysosomal systems, or binding to specific and soluble ligands such as metallothioneins. The later are heat stable and characterised by a high content of cysteine. The quantification of $\mathrm{Fe}, \mathrm{Zn}, \mathrm{Cu}, \mathrm{Mn}, \mathrm{Cd}, \mathrm{Ag}, \mathrm{Ba}$ and $\mathrm{Sr}$ in the tissues of the vent mussel Bathymodiolus $s p$. indicates a high accumulation of these metals in two target organs, the gill and the digestive gland. This accumulation is in relation with high concentrations of metallothioneins, but the subcellular distribution of metals indicates a higher contribution of the insoluble compartment for detoxification processes. These results are compared with the literature data about metal bioaccumlation in Bathymodiolus thermophilus collected at the Galapagos Rift. () Elsevier, Paris
\end{abstract}

bioaccumulation / metallothioneins / lysosomes / toxicity / hydrothermal

Résumé - L'environnement hydrothermal est caractérisé, entre autres, par une concentration élevée en métaux, due à la circulation convective de l'eau de mer dans la croûte océanique et à son interaction avec les roches volcaniques. Les organismes vivant à proximité des sources chaudes s'adaptent à cette richesse en métaux sous forme d'ions ou de sels en suspension, soit en maintenant constante leur teneur interne par des processus d'excrétion, soit en les accumulant sous des formes non toxiques. Différents procédés de détoxication ont été mis en évidence chez certaines espèces hydrothermales : immobilisation et précipitation du métal sous forme insoluble, dans les systèmes lysosomiaux, ou fixation à des ligands spécifiques et solubles dont les plus importants sont les métallothionéines, riches en cystéine et stables à la chaleur. Les teneurs en Fe, $\mathrm{Zn}, \mathrm{Cu}, \mathrm{Mn}, \mathrm{Cd}, \mathrm{Ag}$, Ba et Sr dans les tissus de Bathymodiolus sp., espèce dont la physiologie est encore mal connue, montrent une accumulation importante dans les deux organes cibles, la branchie et la glande digestive. Cette accumulation est associée à des teneurs élevées en métallothionéines, mais la répartition des métaux montre une contribution plus importante du compartiment insoluble, dans les mécanismes de détoxication. Ces résultats sont comparés avec les données de la littérature concernant la bioaccumulation des métaux chez Bathymodiolus thermophilus des Galapagos. (C) Elsevier, Paris

bio-accumulation / métallothionéines / lysosomes / toxicité / hydrothermal 


\section{INTRODUCTION}

Depuis leur découverte en 1977, sur la dorsale des Galapagos $[8,22]$, les peuplements d'invertébrés associés aux sources hydrothermales ont suscité un intérêt particulier, notamment en raison de la toxicité de ce milieu. L'exploration des dorsales océaniques dans tout le Pacifique, et plus récemment dans l'Atlantique, a montré la généralité de ces peuplements dans tous les sites hydrothermaux. Les mollusques bivalves, mytilidae et vesicomyidae, y forment un groupe bien représenté $[20,23]$. Des observations en microscopie électronique ont mis en évidence, chez ces organismes, une association symbiotique, révélée par la présence de bactéries autotrophes à l'intérieur des cellules branchiales $[3,16,21]$.

Les organismes vivant autour des sources chaudes sont soumis à un environnement caractérisé par une combinaison de facteurs inhabituels, tels que des températures élevées et une richesse exceptionnelle en métaux, sous des formes biologiquement assimilables [9]. La survie des individus, en particulier les sédentaires tels que le mollusque bivalve Bathymodiolus, dépend de leur capacité à limiter leurs teneurs internes en éléments ou à accumuler ces éléments sous des formes non toxiques. Il y a peu de données sur l'accumulation des métaux chez les mollusques bivalves hydrothermaux. La toxicité du métal peut être liée à sa teneur ou à sa forme chimique $[13] ; \Gamma \mathrm{e}, \mathrm{Zn}$, $\mathrm{Cu}$ et $\mathrm{Mn}$ sont connus comme biologiquement actifs à faibles concentrations et ils sont essentiels pour la croissance et le métabolisme de ces organismes; en excès, ces métaux peuvent devenir toxiques. $\mathrm{Cd}, \mathrm{Ag}, \mathrm{Ba}$ et $\mathrm{Sr}$ sont connus pour être très toxiques et ne semblent pas impliqués dans le métabolisme de base de ces organismes.

Les mollusques bivalves hydrothermaux développent plusicurs mécanismes de détoxication des métaux :

1) immobilisation et précipitation (sous forme insoluble) dans des inclusions paracristallines et dans des granules inertes, souvent concentrés à l'intérieur des systèmes lysosomiaux [31]; de tels mécanismes ont été mis en évidence chez Bathymodiolus et Calyptogena, lors de travaux préliminaires [4-6, 17] ;

2) fixation à des ligands spécifiques et solubles, dont les plus importants sont les métallothionéines (MT). Ces molécules font partie d'une classe de protéines, aux caractéristiques bien particulières : elles sont solubles, stables à la chaleur, de faible poids moléculaire $(6000-$ $7000 \mathrm{Da}$ chez les mammiferes), riches en cystéine (un tiers des 61 acides aminés totaux) et en groupements sulfhydryles, et ne possèdent ni acide aminé aromatique, ni histidine, ni méthionine [11, 28]. La structure primaire de la protéine est caractérisée par une distribution très déterminée des résidus cystéiques, comme le groupement métal-thiolate : Cys-X-Cys. Ces protéines ont donc une grande capacité de liaison avec certains métaux, comme $\mathrm{Zn}, \mathrm{Cu}, \mathrm{Cd}, \mathrm{Ag}$ et $\mathrm{Hg}$. Toutes les métalloprotéines ainsi caractérisées sont appelées des métallothionéines [1,31].

Les résultats présentés dans ce travail sont des données originales sur la bioaccumulation d'éléments ( $\mathrm{Fe}, \mathrm{Zn}, \mathrm{Cu}$, $\mathrm{Mn}, \mathrm{Cd}, \mathrm{Ag}, \mathrm{Ba}$ et $\mathrm{Sr}$ ) chez le bivalve Bathymodiolus $s p$. de la ride médio-Atlantique (site Menez-Gwen $800 \mathrm{~m}$ et site Lucky-Strike $1700 \mathrm{~m}$ ). Ils sont comparés aux résultats de Smith et Flegal [29], obtenus sur un site hydrothermal de la Dorsale des Galapagos. Les protéines de type métallothionéines ont également été quantifiées dans différents tissus de cette espèce en précisant la répartition de certains métaux ( $\mathrm{Cu}, \mathrm{Zn}$ et $\mathrm{Cd}$ ) entre les ligands intracellulaires insolubles et solubles.

\section{MATÉRIEL ET MÉTHODES}

Les échantillons analysés ont été prélevés dans la zone des Açores (dorsale Atlantique), lors de la mission DIVA 1994, sur dcux sites hydrothermaux de profondeurs différentes : Menez-Gwen $\left(37^{\circ} 0^{\prime} 30^{\prime \prime} \mathrm{N}\right)$ à $800 \mathrm{~m}$ et LuckyStrike $\left(37^{\circ} 17^{\prime} 30^{\prime \prime} \mathrm{N}\right)$ à $1700 \mathrm{~m}$. Sur les deux sites, les échantillons proviennent d'une même plongée, Di 15 pour Menez-Gwen et Di 18 pour Lucky-Strike.

Les peuplements de ces deux sites sont dominés par une espèce nouvelle de moule (genre Bathymodiolus), actuellement en cours de description. Les échantillons ont été disséqués à bord, congelés dans l'azote liquide, puis trois types de tissus (le manteau, la branchie et la glande digestive) lyophilisés et conservés au congélateur jusqu'à l'analyse au laboratoire.

Les résultats obtenus sur une série de moules littorales (type Mytilus) prélevées en milieu sain (Méditerranée) ont été utilisés comme référence.

\subsection{Dosages globaux des métaux dans les lissus des organismes}

Les tissus biologiques, dissous par une attaque à l'acide nitrique concentré et une évaporation à $150^{\circ} \mathrm{C}$, sont analysés en spectrométrie d'absorption atomique électrothermique (SAAE) pour le $\mathrm{Cd}$ et $\mathrm{Ag}$ et en spectrométrie d'émission plasma (ICP-AES) pour $\mathrm{Fe}, \mathrm{Zn}, \mathrm{Cu}, \mathrm{Mn}, \mathrm{Ba}$ et $\mathrm{Sr}$. 
Des blancs d'attaque, analysés avec les échantillons, permettent de détecter d'éventuelles contaminations. Les résultats sont donnés en poids de métal par poids de tissu sec.

\subsection{Dosage des métallothionéines et compartimenta- tion des métaux $\mathrm{Zn}$, Cu et $\mathrm{Cd}$}

Les échantillons sont pesés, puis homogénéisés par hrnyage mécanique dans du tampon TRIS-HCl $(20 \mathrm{mM}$, $\mathrm{pH} 8,6$ ), auquel ont été ajoutés différents inhibiteurs de protéases (aprotinine, leupeptine, benzamidine) et du $\beta$ mercaptoéthanol. Les protéines ainsi mises en solution dans les échantillons sont centrifugées pendant une heure $(10000 \mathrm{~g})$; les composés solubles du surnageant (S1) sont récoltés, dénaturés à la chaleur $\left(75^{\circ} \mathrm{C}\right.$ pendant $15 \mathrm{~min}$ ), puis recentrifugés $(10000 \mathrm{~g}$ pendant $10 \mathrm{~min}$ ) (figure 1). Cette manipulation permet d'isoler les composés thermorésistants, dont les métallothionéines (surnageant S2).

Dans les culots (C1) issus de la première centrifugation, les métaux présents dans les tissus sous des formes inso- lubles sont analysés en spectrométrie d'absorption atomique en flamme ( $\mathrm{S} \Lambda \Lambda \mathrm{F})$ ou électrothermique $(\mathrm{S} \Lambda \Lambda \mathrm{E})$, après attaque par l'acide nitrique $65 \%$.

Les surnageants $\mathrm{S} 2$ permettent, d'une part, la quantification des métallothionéines par polarographie à impulsions différentielles (DPP) [30], en utilisant la technique des ajouts dosés et, d'autre part, l'analyse par SAAF et SAAE des métaux liés aux composés thermostables ( $\mathrm{Zn}$, $\mathrm{Cu}$ et $\mathrm{Cd})$.

\section{RÉSULTATS}

\subsection{Bioaccumulation des métaux dans les tissus des organismes}

Les manteaux présentent des teneurs en métaux souvent plus faibles que celles observées dans les branchies et les glandes digestives (tableau I, figures 2 et 3 ). Le plus souvent, les teneurs sont plus importantes dans les branchies. Les métaux les plus abondants sont $\mathrm{Fe}$ dans les glandes digestives et $\mathrm{Zn}$ et $\mathrm{Cu}$ dans les branchies. La teneur en

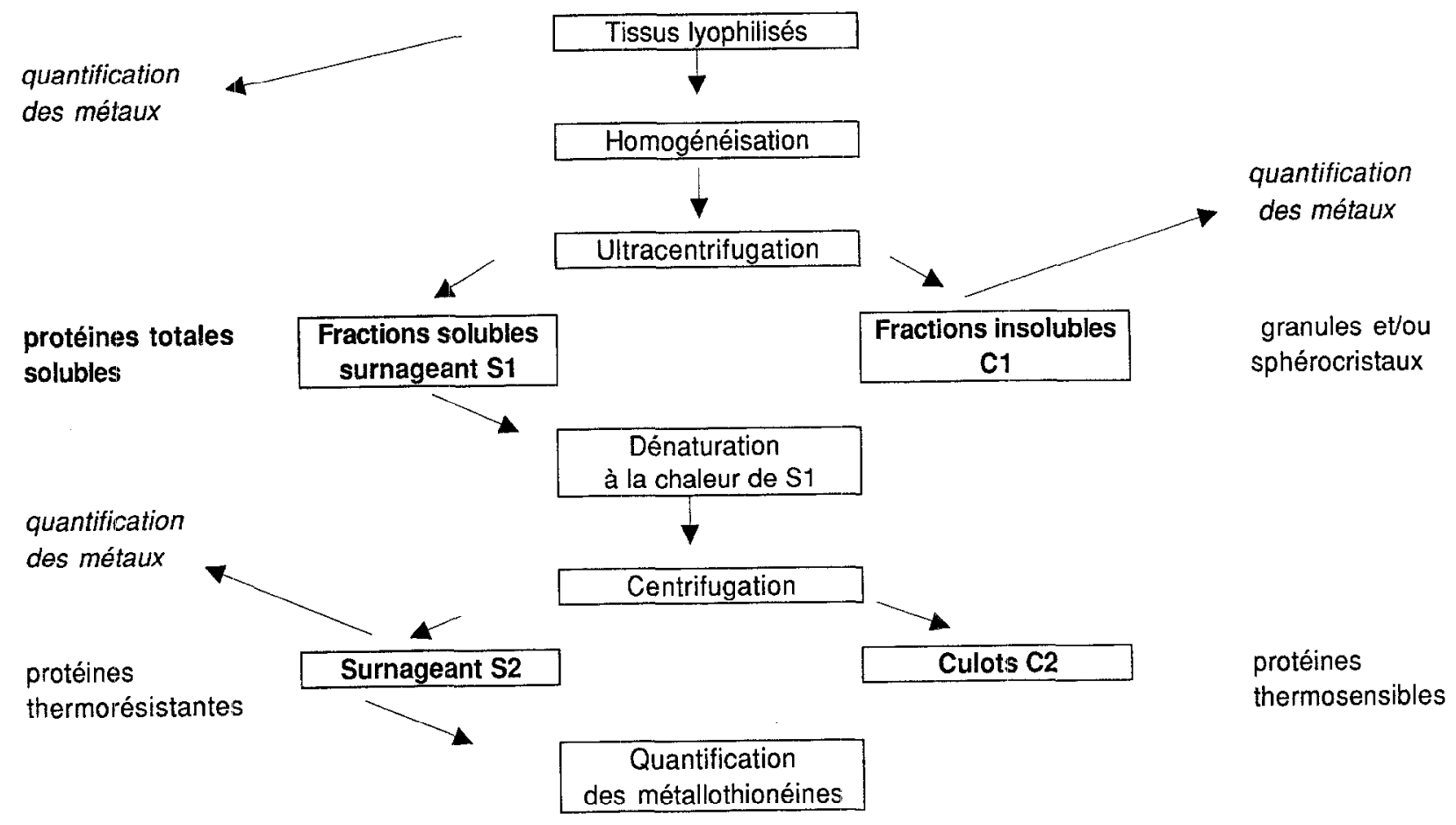

Figure 1. Méthodologie de l'étude de la bioaccumulation des métaux dans les tissus de Rathymodiolus sp.

Figure 1. Methodology used for the study of elemental bioaccumulation within the mollusc Bathymodiolus $\mathrm{sp}$. 
Tableau I. Concentrations en métaux dans les tissus de Bathymodiolus sp. sur les deux sites Açores (Dorsale Atlantique).

Table I. Metal within Bathymodiolus sp. tissues from two Açores sites (Atlantic Rise).

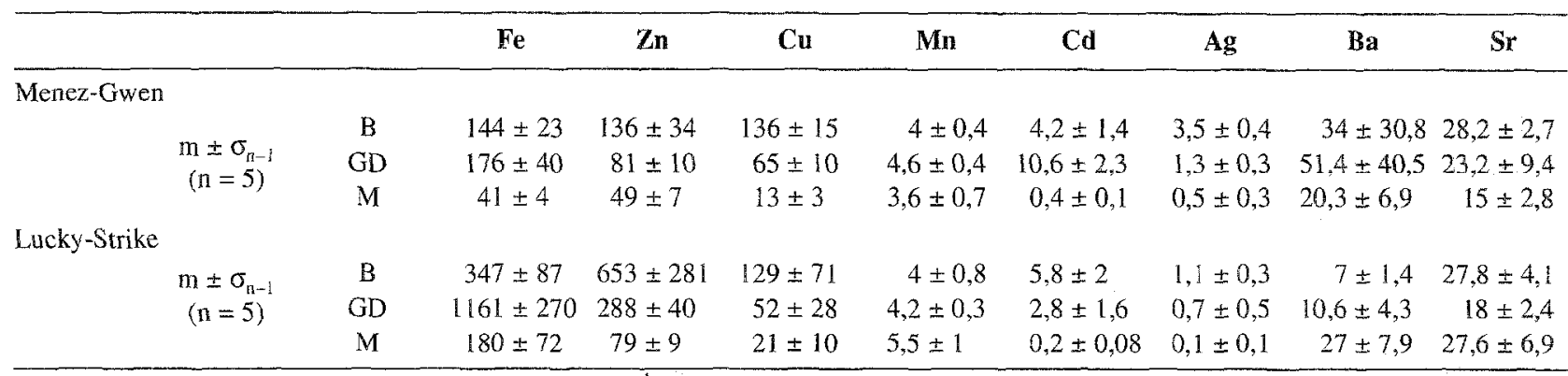

Les teneurs en métal du tissu sec sont exprimées en $\mu \mathrm{g} \mathrm{g}^{-1} . \mathrm{m}:$ moyenne ; $\sigma_{\mathrm{n}-1}$ : écart type ; $:$ branchies ; GD : glandes digestives ; $\mathrm{M}:$ man tcaux.

Concentrations in dry weight are given as $\mu \mathrm{g} \mathrm{g}^{-1} . \mathrm{m}$ : mean; $\sigma_{\mathrm{n}-1}$ : standard deviation. B: gills; GD: digestive gland; $\mathrm{M}$ : mantle.

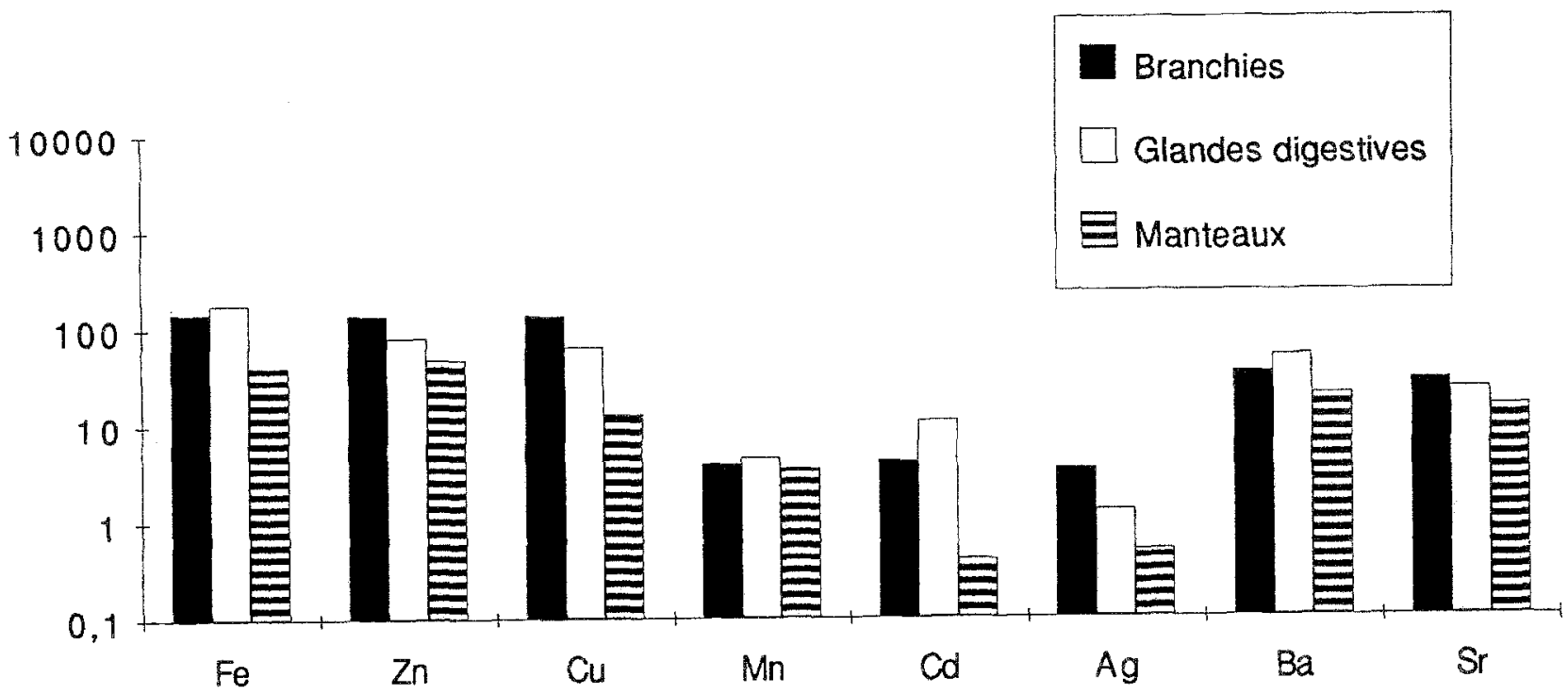

Figure 2. Bathynodiolus sp. Teneurs en métaux dans le tissu sec $\left(\mu \mathrm{g} \mathrm{g}^{-1}\right)$ des moules du site Menez-Gwen.

Figure 2. Bathymodiolus sp. Heavy metal concentrations in dry weight $\left(\mu \mathrm{g} \mathrm{g}^{-1}\right)$ within mussels from Menez-Gwen.

Mn est quasiment constante (entre 3 et $5 \mu \mathrm{g} \mathrm{g}^{-1}$ de tissu $\mathrm{sec})$, quel que soit le tissu. Les teneurs en $\mathrm{Cd}$ sont en général supérieures à celles de $\mathrm{Ag}$. Les teneurs en $\mathrm{Ba}$ sont très variables d'un site à l'autre. Sr présente la même constance que $\mathrm{Mn}$, avec des valeurs plus élevées.

Les teneurs en $\mathrm{Fe}$ et $\mathrm{Zn}$ sont beaucoup plus importantes dans les tissus des organismes du site Lucky-Strike, avec en particulier des valeurs très élevées pour $\mathrm{Fe}$ dans les glandes digestives (1500 $\mu \mathrm{g} \mathrm{g}^{-1}$ de tissu sec). Cd et $\mathrm{Ag}$ s'accumulent davantage dans les branchies ou les glandes digestives, selon le site considéré. Les teneurs en Ba sont du même ordre dans les tissus des organismes du site Lucky-Strike, avec une moyenne un peu plus élevée dans les manteaux. Elles sont globalement plus élevées dans les organismes du site Menez-Gwen, avec des teneurs importantes dans les glandes digestives et faibles dans les manteaux. Pour ce site, la variabilité entre individus est assez grande pour un tissu donné, en particulier dans les 


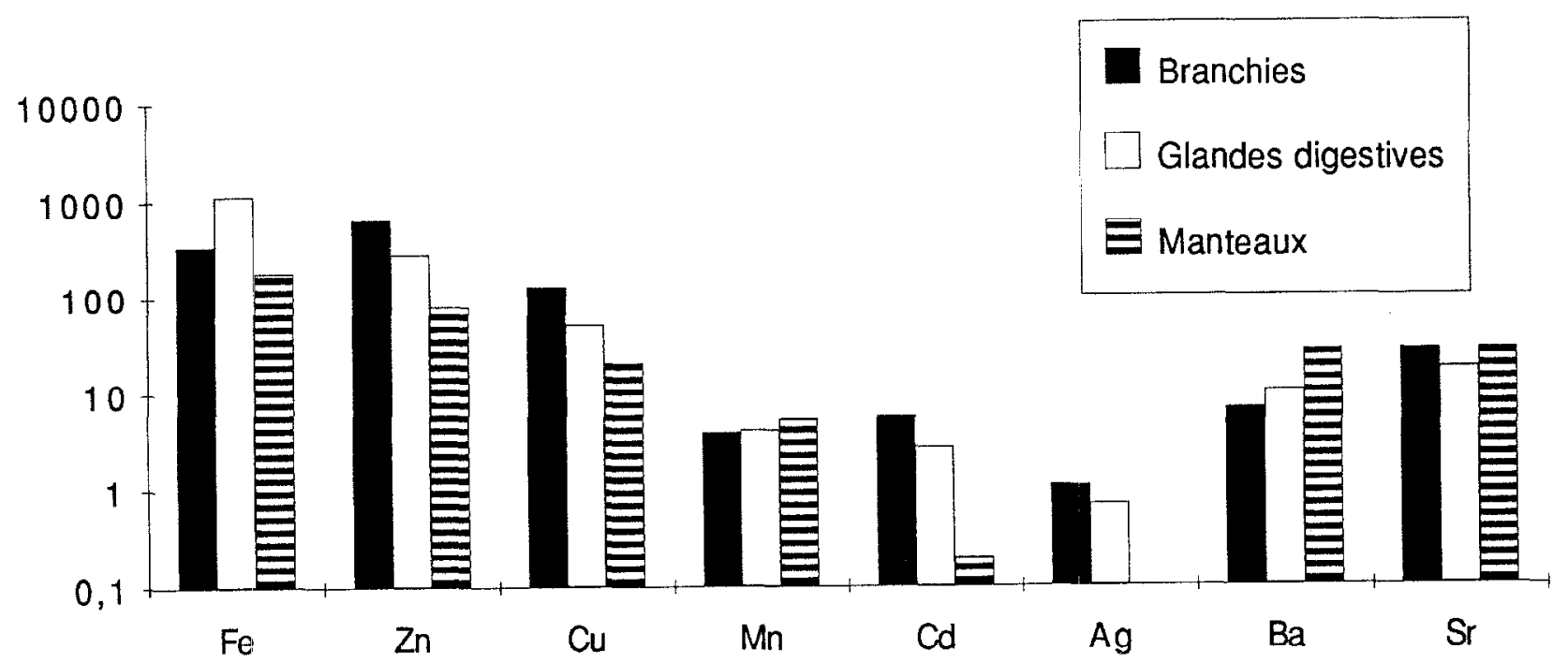

Figure 3. Bathymodiolus sp. Teneurs en métaux dans le tissu sec $\left(\mu \mathrm{g} \mathrm{g}^{-1}\right)$ des moules du site Lucky-Strike.

Figure 3. Bathymodiolus sp. Heavy metal concentrations in dry weight $\left(\mu \mathrm{g} \mathrm{g}^{-1}\right)$ within mussels from Lucky-Strike.

glandes digestives. Les valeurs moyennes (tableau I) ont été calculées en retirant ces valeurs singulières que l'on peut attribuer à l'hétérogénéité du tissu. Les teneurs extrêmes non prises en compte dans ces calculs concernent en particulier Fe $\left(2884 \mu \mathrm{g} \mathrm{g}^{-1}\right), \mathrm{Cu}\left(170 \mu \mathrm{g} \mathrm{g}^{-1}\right)$ et $\operatorname{Ag}\left(10,7 \mu \mathrm{g} \mathrm{g}^{1}\right)$ dans la glande digestive d'un seul individu.

Certains coefficients de variation peuvent rester élevés dans le cas de $\mathrm{Cd}$ et $\mathrm{Ag}$, en raison de teneurs très faibles pour l'ensemble des individus analysés.

\subsection{Analyse des métallothionéines}

De grandes quantités de métallothionéines sont observées dans tous les organes étudiés (branchies et manteaux) de tous les individus des deux sites (tableau II). Les teneurs en métallothionéines sont, d'une manière générale, trois fois plus élevées dans les branchies (environ $3500 \mathrm{\mu g} \mathrm{g}^{-1}$ de tissu sec) que dans les manteaux (environ $1000 \mu \mathrm{g} \mathrm{g}^{-1}$ de tissu sec) et du même ordre dans les organismes des deux sites.

\subsection{Répartition des éléments métalliques $\mathrm{Zn}, \mathrm{Cu}$ et Cd}

La répartition dans un tissu, entre fraction insoluble et fraction soluble, des métaux susceptibles d'être liés à des protéines, varie en fonction du site (tableau III). Pour les
Tableau II. Teneurs en métallothionéines dans les tissus de Bathymodiolus sp. sur les deux sites "Açores".

Table II. Metallothioneins within Bathymodiolus sp. tissues from two Açores sites.

\begin{tabular}{cccc}
\hline & & Branchies & Manteaux \\
\hline $\begin{array}{c}n=5 \\
\begin{array}{c}\text { Mene } z \text {-Gwen } \\
800 \mathrm{~m}\end{array}\end{array}$ & $\mathrm{~m} \pm \sigma_{\mathrm{n}-1}$ & $3824 \pm 794$ & $842 \pm 143$ \\
$\begin{array}{c}\text { Lucky-Strike } \\
1700 \mathrm{~m}\end{array}$ & $\mathrm{~m} \pm \sigma_{\mathrm{n}} \mathrm{l}$ & $3418 \pm 342$ & $1490 \pm 209$ \\
& & &
\end{tabular}

Les teneurs du tissu sec sont exprimées en $\mu \mathrm{g} \mathrm{\textrm {g } ^ { - 1 }}$. $\mathrm{m}$ : moyenne ; $\sigma_{n-1}:$ écart type.

Concentrations in dry weight are given as $\mu \mathrm{g} \mathrm{g}^{-1}$. m: mean; $\sigma_{\mathrm{n}-1}:$ standard deviation.

organismes du site Menez-Gwen, les métaux se retrouvent en majorité dans la fraction soluble, sauf dans le cas du $\mathrm{Cu}$ accumulé dans les branchies. Inversement, dans les organismes du site Lucky-Strike les métaux sont plus localisés dans lcs composćs insolubles, avec en particulier la totalité du $\mathrm{Cu}$ analysé dans les tissus.

La répartition des métaux au sein de la fraction soluble, entre les composés thermostables (dont les métallothionéines) et les composés thermolabiles, diffère là aussi entre les organismes des deux sites (tableau III). Au site Menez-Gwen, Cd est majoritairement associé aux composés thermolabiles dans les deux organes. $\mathrm{Zn}$ est fixé par 
Tableau III. Résultats de l'étude de la répartition des métaux $\mathrm{Zn}$, Cu et Cd entre les différentes fractions.

Table III. Distribution of metals $\mathrm{Zn}, \mathrm{Cu}$ and $\mathrm{Co}$ between the different fractions.

\begin{tabular}{|c|c|c|c|c|c|c|c|c|c|c|c|c|c|}
\hline & & \multicolumn{4}{|c|}{$\mathbf{Z n}$} & \multicolumn{4}{|c|}{$\mathrm{Cu}$} & \multicolumn{4}{|c|}{$\mathrm{Cd}$} \\
\hline & & FI & $\mathbf{F S}$ & CTS & CTL & FI & FS & CTS & CTL & FI & FS & CTS & CTL \\
\hline \multirow[t]{2}{*}{ MG } & Branchies & $32 \%$ & $68 \%$ & $60 \%$ & $40 \%$ & $70,5 \%$ & $29,5 \%$ & $50 \%$ & $50 \%$ & $43 \%$ & $57 \%$ & $29 \%$ & $71 \%$ \\
\hline & Manteaux & $35 \%$ & $65 \%$ & $34 \%$ & $66 \%$ & $31 \%$ & $69 \%$ & $<50 \%$ & $>50 \%$ & $25 \%$ & $75 \%$ & $13 \%$ & $87 \%$ \\
\hline \multirow[t]{2}{*}{ LS } & Branchies & $72 \%$ & $28 \%$ & $31 \%$ & $69 \%$ & $100 \%$ & $0 \%$ & $0 \%$ & $0 \%$ & $74 \%$ & $26 \%$ & $53 \%$ & $47 \%$ \\
\hline & Manteaux & $82 \%$ & $18 \%$ & $100 \%$ & $0 \%$ & $100 \%$ & $0 \%$ & $0 \%$ & $0 \%$ & $100 \%$ & $0 \%$ & $0 \%$ & $0 \%$ \\
\hline
\end{tabular}

MG : site Menez-Gwen; LK: site Lucky-Strike. HI et FS: pourcentage de métaux associés respectivement à la fraction insoluble (granules ou sphérocristaux) et à la fraction fraction soluble, après la première centrifugation. CTS et CTL: pourcentage de métaux de la fraction soluble (FS) associés respectivement aux composés thermo-stables et aux composés thermo-labiles.

MG: Menez-Gwen; LK: Lucky-Strike. FI and FS: percentage of metals respectively associated with the insoluble fraction and the soluble fraction, resulting from the first centrifugation. CTS and CTL: percentage of soluble fraction metals respectively associated with heat-stable compounds and heat-denaturable proteins.

les composés solubles thermostables dans les branchies, et $\mathrm{Cu}$ se répartit de manière assez équivalente entre les deux types de composés, quel que soit l'organe considéré. Pour les organismes du site Lucky-Strike, Zn, dans les manteaux, semble lié en totalité aux composés solubles thermostables, mais dans les branchies, il est en majorité dans les composés thermolabiles. Enfin, $\mathrm{Cd}$ se retrouve en proportion quasiment égale entre les deux types de composés.

\section{DISCUSSION}

\subsection{Bioaccumulation des métaux}

La principale interface entre les organismes bivalves des sources hydrothermales et leur environnement se situe dans les branchies où les métaux, sous forme dissoute, peuvent pénétrer par des phénomènes diffusifs [9]. De plus, contrairement aux vesicomyidae, les mytilidés profonds possèdent un tube digestif fonctionnel $[18,19]$ et la filtration des particules par voie digestive constitue un moyen supplémentaire pour l'entrée des métaux dans l'organisme.

D'une manière générale, les teneurs en métaux sont faibles dans les manteaux, par rapport aux autres tissus. Cette accumulation réduite peut s'expliquer par le rôle plutôt passif du manteau vis-à-vis de l'environnement, puisqu'il sert essentiellement à l'accumulation de réserves et à la sécrétion de la coquille $[13,27]$. Seul Ba fait exception et il semble être un métal plus lié au manteau, dans le cas des organismes du site Lucky-Strike. Bien qu'entrant dans la composition de la coquille de ces bivalves, $\mathrm{Ba}$ n'est pas connu comme biologiquement actif. Chimiquement, il est très proche de $\mathrm{Sr}$ or, dans ces organismes, les comportements respectifs des deux éléments semblent bien différents; les teneurs en Ba sont très variables, alors que les concentrations en $\mathrm{Sr}$ sont quasiment constantes. Il semblerait que $\mathrm{Ba}$ ait une fonction particulière dans ces organismes. L'inclusion de baryum dans le carbonate des coquilles leur donne une meilleure stabilité chimique à des profondeurs où les carbonates peuvent devenir solubles.

Les teneurs en métaux dans les branchies, où siège l'activité symbiotique $[16,21]$, sont souvent les plus élevées. $\mathrm{Fe}$ (et pour partie $\mathrm{Ba}$ ) est toujours plus concentré dans les glandes digestives. Cette accumulation préférentielle pourrait être en relation avec des procédés de détoxication [4-7, 9]. L'accumulation de $\mathrm{Fe}$ dans les glandes digestives des organismes pourrait être expliquée par la présence de particules minérales dans le milieu environnant, probablement de la pyrite, sur lesquelles les métaux peuvent s'adsorber. En effet, des particules minérales ont été observées dans la glande digestive chez Bathymodiolus de l'EPR (East Pacific Rise) à $13^{\circ} \mathrm{N}[5,6]$.

Dans le cas d'accumulation relative de Ba dans la glande digestive, la formation de $\mathrm{BaSO}_{4}$ pourrait jouer un rôle de détoxication vis à vis du soufre, ou représenter une accumulation particulaire, due au milieu hydrothermal. La variabilité de certaines teneurs en métaux déjà citée est probablement à mettre en relation avec ce phénomène qui introduit une hétérogénéité dans les glandes digestives.

Comme pour les espèces littorales $[9,13]$, la phase dissoute, dans les branchies, et la phase particulaire dans les glandes digestives, sont les deux sources potentielles de métaux pour les moules hydrothermales. De plus, l'accumulation sélective de métal dans un tissu semble liée à la 
forme chimique du métal et aux mécanismes de détoxication associés au tissu. La figure 4 résume les mécanismes d'accumulation et de détoxication des métaux chez Bathymodiolus $s p$. Ces métaux peuvent être également impliqués dans des processus biologiques liés à la symbiose et au fonctionnement particulier de ces organismes profonds.

La comparaison entre les sites hydrothermaux montre dans chaque tissu un rapport entre la concentration à $1700 \mathrm{~m}$ et la concentration à $800 \mathrm{~m}$ toujours supérieur à l'unité pour $\mathrm{Fe}$ et $\mathrm{Zn}$, avec une valeur de 2 à 5 pour les branchies, de 3 à 7 pour les glandes digestives et de 2 à 4 pour les manteaux. La plus forte accumulation de ces métaux dans les organismes du site Iucky-Strike pourrait être due soit à leurs concentrations plus importantes dans le milieu environnant, soit à leur présence sous des formes chimiques plus facilement assimilables par les organismes.

Les teneurs en $\mathrm{Cu}, \mathrm{Mn}$ et $\mathrm{Sr}$ sont assez semblables dans les organismes des deux sites, soit parce qu'elles sont identiques dans le fluide, soit parce que les organismes régulent leurs teneurs par des phénomènes d'excrétion, en rapport avec leur fonction biologique connue. Les faibles teneurs en $\mathrm{Cd}$ et $\mathrm{Ag}$, par rapport aux autres métaux peuvent s'expliquer par des concentrations relatives également plus faibles dans le fluide.

Les concentrations en métaux ont été mesurées chez Bathymodiolus thermophilus de la ride des Galapagos (tableau IV). Les métaux n'ont pas été analysés dans le manteau, qui n'est pas considéré comme un organe cible pour la bioaccumulation [29]. De même, $\mathrm{Cd}$ et Ba n'apparaissent pas dans cette étude ; dans le fluide émis sur le site des Galapagos les concentrations en $\mathrm{Cd}$ sont presque nulles et, dans les organismes, les teneurs sont proches de celles de l'eau de mer. Ba semble être un métal plus lié au manteau (d'après les teneurs dans les moules de LuckyStryke), ce qui expliquerait pourquoi il n'a pas été analysé. Les teneurs en $\mathrm{Fe}$ chez les organismes des Galapagos sont proches de celles trouvées chez les organismes du site Lucky-Strike avec, là aussi, des teneurs dans les glan-

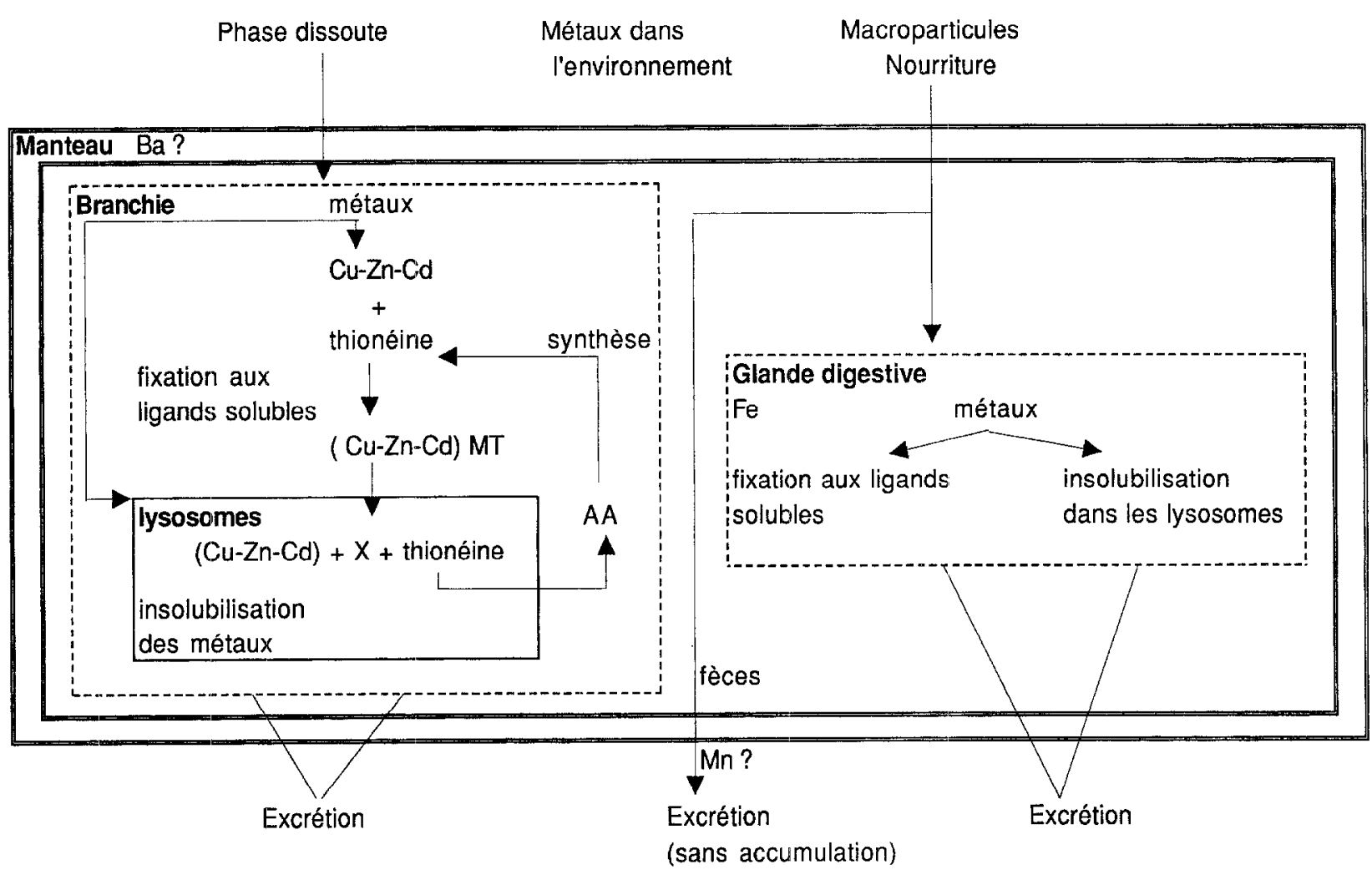

Figure 4. Bilan de l'accumulation et la détoxication des métaux chex Bathymodiolus sp. MT: métallothionéines; AA: acides aminés.

Figure 4. Mechanisms of metal accumulation and detoxification within Bathymodiolus sp. MT: metallothioneins; AA: amino acid. 
Tableau IV. Teneurs en éléments traces des moliusques hydrothermaux Bathynodiolus thermophilus [29] et Calyptogena magnifica [26] et du vestinientifère Riftia pachyptila [10].

Table IV. Concentrations of selected metals in hydrothermal molluses Bathymodiolus thermophilus [29] and Calyptogena magnifica [26] and in the vestimentiferan Riftia pachyptila [10].

\begin{tabular}{|c|c|c|c|c|c|c|c|c|c|}
\hline & $\mathrm{Fe}$ & $\mathbf{Z n}$ & $\mathrm{Cu}$ & Mn & $\mathrm{Cd}$ & $\mathrm{Ag}$ & $\mathrm{Ba}$ & $\mathrm{Sr}$ & As \\
\hline \multicolumn{10}{|l|}{ Galapagos $(n=4)$} \\
\hline \multicolumn{10}{|c|}{ Bathymodiolus thermophilus } \\
\hline Branchies & $310 \pm 38,1$ & $217 \pm 279$ & $41,3 \pm 34,2$ & $111 \pm 131$ & NA & $51,8 \pm 49,6$ & NA & $118 \pm 98,8$ & $36,5 \pm 49,1$ \\
\hline Glandes digestives & $948 \pm 281$ & $60,9 \pm 40,4$ & $30 \pm 25,9$ & $25,8 \pm 17,2$ & NA & $21,4 \pm 8,49$ & NA & NA & $23,2 \pm 5,34$ \\
\hline \multicolumn{10}{|l|}{$13^{\circ} \mathrm{N}$} \\
\hline Branchies & 389 & 369 & 25 & 7,9 & 0,6 & 1 & NA & NA & 15 \\
\hline \multicolumn{10}{|c|}{ Calyptogena magnifica } \\
\hline Branchies & $1931 \pm 626$ & $1560 \pm 540$ & $219 \pm 102$ & $<7$ & $46 \pm 12$ & $46,5 \pm 28$ & $<12$ & $32,7 \pm 12,5$ & $33,2 \pm 16$ \\
\hline Manteaux & $302 \pm 274$ & $1772 \pm 1088$ & $150 \pm 7,2$ & $13,3 \pm 2,4$ & $1,2 \pm 0,4$ & $50,7 \pm 7$ & 85 & $75,2 \pm 15$ & $28,2 \pm 1,8$ \\
\hline
\end{tabular}

Les teneurs en métal dans le tissu sec sont exprimées en $\mu \mathrm{g} \mathrm{g}{ }^{-1}$ (moyenne \pm écart type). B: branchies; GD: glandes digestives. NA: non analysé.

Concentrations in dry weight are given as $\mu \mathrm{g} \mathrm{g}^{-1}$ (mean \pm standard deviation). B: gills; GD: digestive gland. NA: not analysed.

des digestives bien supérieures à celles des branchies. Les moules de ces deux sites sont donc marquées par une accumulation importante de particules riches en métaux, par voie digestive. Les teneurs en $\mathrm{Zn}$ sont du même ordre que dans le site Menez-Gwen ; elles sont globalement plus importantes dans les glandes digestives des organismes des sites Açores, probablement à cause d'une richesse moindre de ce métal sous forme particulaire dans l'environnement hydrothermal des Galapagos. Les teneurs en métaux essentiels ( $\mathrm{Cu}, \mathrm{Zn}$ ) dans les tissus, et plus particulièrement dans un organe comme la glande digestive (très impliquée dans le métabolisme du bivalve), varient beaucoup en fonction des besoins physiologiques, euxmêmes liés aux fonctions essentielles (croissance, reproduction...). Les concentrations en $\mathrm{Mn}$, Ag et $\mathrm{Sr}$ sont beaucoup plus élevées dans les moules des Galapagos. Les eaux des Galapagos étant assez froides et contenant peu de métaux, mis à part $\mathrm{Mn}\left(\right.$ de l'ordre de $32000 \mu \mathrm{g} \mathrm{L}^{-1}$ ), les organismes filtrent peut-être des quantités d'eau plus importantes pour leurs besoins physiologiques, entraînant ainsi une accumulation relative de $\mathrm{Mn}$ el de fortes concentrations en Mn dans les tissus.

Des dosages de métaux ont également été réalisés chez d'autres espèces hydrothermales, telles Riftia pachyptila [10] et Calyptogena magnifica [26] (tableau IV) prélevés au niveau de l'EPR. Les teneurs sont très différentes entre ces deux organismes et les moules, Bathymodiolus termophilus des Galapagos et Bathymodiolus de la dorsale atlantique. Les variations sont observées aussi bien entre des organismes du même type vivant dans des sites différents qu'entre des organismes différents vivant dans une même région.

La comparaison des teneurs en métaux des espèces hydrothermales et de l'espèce littorale Mytilus edulis ( tableau $V$ ) met en évidence une plus forte accumulation en $\mathrm{Fe}, \mathrm{Zn}, \mathrm{Cu}, \mathrm{Cd}$ et $\mathrm{Ba}$ chez les moules symbiotiques (à l'exception de $\mathrm{Fe}$ au site Menez-Gwen). Cette différence avec le milieu côtier peut être en relation directe avec les concentrations en éléments traces dans les flui-

Tableau V. Concentrations en métaux dans les tissus de la moule Mytilus edulis (d'après Ferreiro, données non publiées).

Table V. Metals within coastal Mytilus edulis tissues (according Ferreiro, unpublished data).

\begin{tabular}{|c|c|c|c|c|c|c|c|c|}
\hline $\mathbf{n}=\mathbf{3}$ & & $\mathrm{Fe}$ & $\mathrm{Zn}$ & $\mathrm{Cu}$ & Mn & $\mathrm{Cd}$ & $\mathrm{Ag}$ & $\mathbf{B a}$ \\
\hline Mytilus edulis & Branchies & $203,5 \pm 87,5$ & $97 \pm 26$ & $10 \pm 2$ & $5,4 \pm 1,2$ & $1,1 \pm 0,3$ & $0,6 \pm 0,2$ & $4,1 \pm 1,6$ \\
\hline Méditerranée & Manteaux & $121 \pm 74$ & $61 \pm 13$ & $8 \pm 2$ & $5 \pm 3,1$ & $0,1 \pm 0,2$ & $0,6 \pm 0,5$ & $1,7 \pm 0,7$ \\
\hline
\end{tabular}

Les valeurs de métal dans le tissu sec sont exprimées en $\mu \mathrm{g} \mathrm{g}^{-1}$ (moyenne \pm écart type).

Concentrations in dry weight are given as $\mu \mathrm{g} \mathrm{g}^{-1}$ (mean \pm standard deviation). 
des hydrothermaux, plus élevées que celles généralement observées dans l'eau de mer $[14,15]$. Néanmoins, Fe semble être accumulé en grande quantité dans les organismes du site Menez-Gwen comme chez les moules littorales; il ne correspond sans doute pas à une propriété particulière des mytilidés profonds.

Les teneurs en métaux dans les tissus de moules (Mytilus galloprovincialis) situées dans des zones côtières polluées, telles que la région de Scarlino au Nord de la mer Tyrrhénienne, montrent une accumulation importante en $\mathrm{Fe}, \mathrm{Mn}$ et $\mathrm{Pb}$ [25]. Les teneurs sont notamment très élevées dans les glandes digestives, $4000 \mu \mathrm{g} \mathrm{g}^{-1}$ de tissu sec pour $\mathrm{Fe}, 138 \mu \mathrm{g} \mathrm{g}^{-1}$ de tissu sec pour Mn et $92 \mu \mathrm{g} \mathrm{g}^{-1} \mathrm{de}$ tissu sec pour $\mathrm{Pb}$. Mais les teneurs en $\mathrm{Zn}$ et $\mathrm{Cu}$ restent du même ordre que celles généralement trouvées dans les moules de milieu sain. Des expériences de transplantation en milieu sain montrent une excrétion rapide du $\mathrm{Mn}$ et $\mathrm{Fe}$, un stockage du $\mathrm{Pb}$, et une régulation des teneurs en métaux essentiels, $\mathrm{Cu}$ et $\mathrm{Zn}$ [24] traduisant différents temps d'intégration (bilan entrées/excrétion) pour chaque élément, en fonction du mécanisme de détoxication et/ou d'excrétion employé par les organismes. Les teneurs en $\mathrm{Fe}, \mathrm{Zn}, \mathrm{Cu}$ et $\mathrm{Cd}$ ont également été analysées chez ce type de moule dans la baie d'Augusta en Sicile, caractérisée par des rejets industriels et urbains [2]. Les valeurs observées dans les organismes entiers restent très inférieures à celles des moules hydrothermales. En ce qui concerne les métaux, la principale différence avec les milieux côtiers les plus pollués vient donc du large spectre des apports en éléments toxiques, unique pour les sources hydrothermales.

\subsection{Teneurs en métallothionéines}

Les teneurs en métallothionéines sont plus importantes dans les branchies que dans les manteaux, plutôt passifs vis-à-vis de l'accumulation des métaux [13, 27]. L'accumulation des métaux étant la plus importante dans les branchies (excepté pour $\mathrm{Fe}$ ), on en déduit qu'une exposition permanente à de forts taux de $\mathrm{Zn}, \mathrm{Cu}, \mathrm{Cd}$ et $\mathrm{Ag}$ (métaux pouvant être liés aux métallothionéines) pourrait induire la synthèse de ces protêines, comme dans le cas des organismes côtiers [28]. Les branchies sont également des tissus à fortes concentrations bactériennes, dont la participation aux mécanismes de détoxication, bien qu'hypothétique, a été envisagée [9]. Les mêmes types de bactéries, méthanotrophes et sulfo-oxydantes, observées dans les moules des deux sites Açores, pourraient expliquer les teneurs semblables en métallothionéines dans les organismes de ces sites. Le rôle des bactéries dans les processus de détoxication est encore mal connu et doit être précisé. D'importantes quantités de métallothionéines ont également été déterminées dans la branchie du vestimentifère Riftia pachyptila de la dorsale EstPacifique, autre espèce majeure des communautés hydrothermales qui présente un même type d'association symbiotique avec des bactéries chimiotrophes [12].

\subsection{Répartition des éléments métalliques $\mathrm{Zn}, \mathrm{Cu}$ et Cd}

Les organismes du site Lucky-Strike semblent utiliser préférentiellement l'immobilisation dans les systèmes lysosomiaux, comme procédé de détoxication de $\mathrm{Zn}, \mathrm{Cu}$ et $\mathrm{Cd}$. Dans le cas des organismes du site Menez-Gwen, la quantité de métaux associés aux composés thermostables (dont les métallothionéines) représente une proportion assez faible des métaux présents dans le cytosol (S1), sauf pour $\mathrm{Zn}$ contenu dans les branchies. Les composés thermolabiles, constitués de protéines et de composés plus ou moins complexes, semblent capables de fixer de grandes quantité de mélaux, en parliculier Cd. Les métallothionéines, bien que présentes en grandes quantités dans ces organismes, ne semblent pas avoir un rôle dominant dans la détoxication. Les différences de proportions entre les métaux sont également à mettre en relation avec les phénomènes d'affinité relative de ces protéines pour un métal ou de compétition entre les différents métaux pour le site de fixation $[11,28]$.

A titre de comparaison, notons que la répartition subcellulaire des métaux chez les moules de Scarlino est différente selon l'élément, mais qu'clle est scmblablc entrc lcs organismes : on retrouve l'importance de la fraction insoluble pour le stockage $\mathrm{du} \mathrm{Pb}$, alors que $\mathrm{Zn}$ et $\mathrm{Cu}$ sont liés à des protéines de type métallothionéines, qui régulent leur métabolisme intracellulaire [24].

\section{CONCLUSION}

Les moules hydrothermales du genre Bathymodiolus présentent une capacité importante d'accumulation des métaux, tels que $\mathrm{Fe}, \mathrm{Zn}, \mathrm{Cu}, \mathrm{Cd}, \mathrm{Ag}$, $\mathrm{Ba}$ et $\mathrm{Sr}$, comparable ou supérieure à celle des milieux littoraux les plus pollués. L'analyse des teneurs des tissus a mis en évidence différents organes bioaccumulant ces métaux : les branchies pour les éléments dissous et les glandes digestives pour les éléments sous forme particulaire. De plus, 
Bathymodiolus provenant des deux sites Açores présente une grande singularité par rapport aux organismes de même type vivant dans des environnements différents et par rapport aux autres organismes hydrothermaux. Les teneurs en métaux dans les organismes varient d'un site hydrothermal à l'autre, sans doute en fonction de leur concentration dans le fluide, de leur forme chimique et de l'importance relative des mécanismes d'accumulation et d'excrétion au sein des organismes.

L'activité hydrothermale est une source importante de métaux pour l'océan mondial et la bioaccumulation de ces éléments par les organismes profonds joue un rôle non négligeable dans le cycle global des métaux dans l'océan.

I.es conditions extrêmes des milieux hydrothermaux obligent les organismes à intensifier les mécanismes de détoxication des métaux. Ainsi, des teneurs élevées en métallothionéines ont été trouvées dans les moules des deux sites, surtout dans les branchies. Elles semblent associées principalement au $\mathrm{Zn}$, mais chez les moules
Bathymodiolus sp., $\mathrm{Zn}, \mathrm{Cu}$ et $\mathrm{Cd}$ pourraient se retrouver majoritairement sous des formes insolubles dans les systèmes lysosomiaux ou être associés à des composés thermolabiles, car la fixation des métalix par les métallothionéines ne représente pas un mécanisme de détoxication à long terme.

\section{Remerciements}

Les auteurs remercient les équipages du navire océanographique Nadir et du submersible Nautile (Genavir/ Ifremer), pour la récolte du matériel durant la campagne Diva (1994), financée en partie par le programme Amores (MAST3-CT95-0040), ainsi que A.M. Alayse, chef de mission, et l'ensemble des participants à cette campagne. Contrat Otan $n^{\circ}$ CRG 931052, Ura CNRS 2071. Les auteurs remercient également A.M. de Kersabiec pour l'aide apportée dans l'analyse des échantillons. Ce manuscrit a bénéficié de la lecture attentive de J. Boutler.

\section{RÉFÉRENCES}

[1] Bordin G., Cordeiro Raposo F., MicCourt J, Rodriguez A.R., Identification de métalloprotéines du type métallothionéine chez un mollusque marin bivalve, C. R. Acad. Sci. Paris (1994) 1057-1064.

[2] Castagna A., Sinatra F., Castagna G., Stoli A., Zafarana S., Trace element evaluations in marine organisms, Mar. Poll. Bull. 16 (1985) 416-419.

[3] Cavanaugh C.M., Symbiotic chemoautropic bacteria in marine invertebrate from sulfide-rich habitas, Nature 302 (1983) 58 61.

[4] Chassard-Bouchaud C., Fiala-Médioni A., Galle P., Étude microanalytique de Bathymodiolus sp. (mollusque Lamelibranche Mytilidae) provenant des sources de la Ride du Pacifique oriental, Données préliminaires, C. R. Acad. Sci. Paris, 302 (1986) 117-124.

[5] Chassard-Bouchaud C., Fiala-Médioni A., Boumati P., Escaig F., Kleinbauer F., Brissard J., Galle P., Calyptogena phaseoliformis (mollusque bivalve) indicateur biologique des phénomènes géochimiques, associés aux zones de subduction situées au large du Japon, C. R. Acad. Sci. Paris 306 (1988a) 237244.

[6] Chassard-Bouchand C., Boumati P., Escaig F., Laubier L., Desbruyères D., Fiala-Médioni A., Outrequin M., Inglebert R.N., Galle P., Microanalyse et hydrothermalisme océanique. Premiers résultats et perspectives dans le domaine de la biologie, Oceanol. Acta (1988b) 203-218.
[7] Childress J.J., Fisher C.R., The biology of hydrothermal vent animals: physiology, biochemistry and autotrophic symbioses, Oceanol. Mar. Biol. Ann. Rev. 30 (1992) 337-441.

[8] Corliss J.B., Dymond J., Gordon L.I., Edmond J.M., Herzen R.P.V., Ballard R.D., Green K., Williams D., Bainbridge A., Crane K., Van Andel T.H., Submarine thermal springs on the Galapagos Rift, Science 203 (1979) 1073-1083.

[9] Cosson-Mannevy M.-A., Cosson R.P., Gaill F., Laubier L., Transfert, accumulation et régulation des éléments minéraux chez les organismes des sources hydrothermales, Oceanol. Acta N. Sp. 8 (1988) 219-226.

[10] Cosson-Mannevy M.A., Cosson R.P., Amiard-Triquet C., Amiard J-C., Métayer C., Elemental bioaccumulation in hydrothermal vent biota on the EPR : organotropism and potential influence of bacteria in: Heavy metals in the environment, (Ed.) by Vernet J.P., CEP Consultants Ltd. Publishers, Edinburgh U.K., 1 (1989) 73-76.

[11] Cosson R., Les métallothionéines, Anal. Mag. 20 (1992) 50 53.

[12] Cosson R., La bioaccumulation des éléments minéraux chez le Vestimentifere Riftia pachyptila, Oceanol. Acta 19 (1996) 163-176.

[13] Dallinger R., Rainbow P.S., Ecotoxicology of metals in invertebrates, Lewis publishers (1992) 461 p.

[14] Edmond J.M., Von Damm K.L., McDuff R.E., Measures C.I., Chemistry of hot springs on the East Pacific Rise and their effluent dispersal, Nature 297 (1982) 187-191. 
[15] Edmond J.M., Von Damm K.L., Chemistry of ridge crest hot springs, Bull. Bioll. Soc. Whas. 6 (1985) 43-47.

[16] Fiala-Médioni A., Mise en évidence par microscopie électronique à transmission de l'abondance de bactéries symbiotiques dans la branchie de mollusques bivalves de sources hydrothermales profondes, C. R. Acad. Sci. Paris 298 (1984) 487-492.

[17] Fiala-Médioni A., Michalsky J.C., Jollès J., Alonso C., Montreuil J., Lysosomic and lysozyme activities in the gill bivalves from deep hydrothermal vents, C. R. Acad. Sci. Paris 317 (1994) 239-244.

[18] Hily A., Le Pennec M., Prieur D., Fiala-Médioni A., Anatomie et structure du tractus digestif d'un Mytilidae des sources hydrothermales profondes de la ride du Pacifique oriental, Cah. Biol. Mar. 27 (1986) 235-241.

[19] Kenk K.C., Wilson B.R., A new mussel (bivalvia, Mytilidae) from hydrothermal vents in the Galápagos Rift zone, Malacologica 26 (1985) 253-271.

[20] Laubier L., Desbruyères D., Les oasis du fond des océans, La Recherche 61 (1984) 1508-1517.

[21] Le Pennec M., Hily A., Anatomie, structure et ultrastructure de la branchie d'un Mytilidae des sites hydrothermaux du Pacifique oriental, Oceanol. Acta 8 (1984) 181-190.

[22] Lonsdale P.F., Clustering of suspending-feeding macrobenthos near abyssal hydrothermal vents at oceanic speading centers, Deep-Sea Res. 24 (1977) 857-863.

[23] Lutz R.A., Kennish M.J., Ecology of deep-sea hydrothermal vent communities: a review, Rev. Geophys. 31 (1993) 211237.
[24] Regoli F., Orlando E., Accumulation and subcellular distribution of metals $(\mathrm{Cu}, \mathrm{Fe}, \mathrm{Mn}, \mathrm{Pb}$ and $\mathrm{Zn}$ ) in the Mediterranean Mussel Mytilus galloprovincialis during a field transplant experiment, Mar. Poll. Bull. 28 (1994) 592-600.

[25] Regoli F., Principato G., Glutathione, glutathione-dependent and antioxidant enzymes in mussel, Mytilus galloprovincialis, exposed to metals under field and laboratory conditions: implications for use of biochemical biomarkers, Aquat. Toxicol. 31 (1995) 143-164.

[26] Roesijadi G., Crecelius E.A., Elemental composition of the hydrothermal vent Calyptogena magnifica from the East Pacific Rise, Mar. Biol. 83 (1984) 155-161.

[27] Roesijadi G., Young J.S., Crecelius E.A., Thomas L.E., Distribution of trace metals in the hydrothermal vent clam, Calyptogena magnifica, Biol. Soc. Wash. Bull. 6 (1985) 311324.

[28] Roesijadi G., Metallothioneins in metal regulation and toxicity in aquatic animals, Aquat. Toxicol. 22 (1992) 81-114.

[29] Smith D.R., Flegal A.R., Elemental concentrations of hydrothermal vent organisms from the Galápagos Rift, Mar. Biol. 102 (1989) 127-133.

[30] Thompson J.A.J., Cosson R., An improved electrochemical method for the quantification of metallothioneins in marine organisms, Mar. Env. Res. 11 (1984) 137-152.

[31] Viarengo A., Nott A., Mechanisms of heavy metal cation homeostasis in marine invertebrates, Comp. Biochem. Physiol. $104 \mathrm{c}(1993)$ 355-372. 